$16^{\text {th }}$ International Congress of Metrology, 04008 (2013)

DOI: $10.1051 /$ metrology/201304008

(C) Owned by the authors, published by EDP Sciences, 2013

\title{
Analysis of a national low dc resistance inter-laboratories comparison
}

\author{
F. Galliana ${ }^{1}$, E. Gasparotto ${ }^{2}$, P. P. Capra ${ }^{3}$ \\ ${ }^{1,2,3}$ National Institute of Metrological Research, (INRIM) str. delle Cacce, 91 - 10135 (TURIN Italy)
}

\begin{abstract}
In this paper the description of an inter-laboratories comparison between the National Institute of Metrological Research (INRIM) and nineteen Italian Secondary Calibration Electrical Laboratories, is given along with its main results in graphical way. The transportable standards were three Tinsley four terminal standard resistors to be calibrated in oil respectively at $5 \mathrm{~A}$ and $20 \mathrm{~A}(1 \mathrm{~m} \Omega)$, $1 \mathrm{~A}$ and $5 \mathrm{~A}(10 \mathrm{~m} \Omega), 0.1 \mathrm{~A}$ and $1 \mathrm{~A}(100 \mathrm{~m} \Omega)$. The comparison results were satisfactory, except only in a case of a Laboratory for which these measurements were outside its accreditation fields.
\end{abstract}

\section{Introduction}

An inter-laboratory comparison aims to verify the compatibility of the measures of two or more Laboratories performed on the same measurand within their measurement uncertainties. Since its start the Italian Calibration Laboratories body (formerly "Servizio di Taratura in Italia" SIT) used to perform bilateral comparisons between the National Metrology Institutes and the Secondary calibration Laboratories to evaluate their technical competence in the framework of their first accreditation or in the periodical renewal of their accreditation. Normally, the results were elaborated according to the ISO Guide 43. Nowadays the number of Secondary Laboratories grew significantly, so the possibility to perform bilateral comparisons became quite difficult. A possible solution for this problem could be the use accredited of Inter-laboratory Comparisons providers (ILCs) [1, 2]. In this occasion the Italian Accreditation body (now ACCREDIA) gave to the National Institute of Metrological Research (INRIM) the task to perform a an inter-laboratories comparison between INRIM and nineteen Italian Secondary calibration Electrical accredited Laboratories in the field of low dc resistance. The transportable standards for the comparison were three Tinsley $\bmod 3504 \mathrm{C} 1 \mathrm{~m} \Omega, 10 \mathrm{~m} \Omega$ and $100 \mathrm{~m} \Omega$ four terminal standard resistors to be calibrated respectively at $5 \mathrm{~A}$ and $20 \mathrm{~A}(1 \mathrm{~m} \Omega), 1 \mathrm{~A}$ and $5 \mathrm{~A}(10 \mathrm{~m} \Omega), 0.1 \mathrm{~A}$ and $1 \mathrm{~A}$. $(100 \mathrm{~m} \Omega)$. The results of this comparison will be utilized by the accreditation body for the evaluation of the measurement competence of the participating Laboratories in the field of low dc resistance. The comparison took place from September 2012 to May 2013. During this period INRIM measured the travelling resistors before, after the circulation, and twice during the circulation.

\section{Participating Laboratories}

The participating Laboratories were:

- ASIT INSTRUMENTS, Orbassano (TO), accredited calibration laboratory (acl) 188;

- ARO, Biassono (MI) acl 46;

- AVIATRONIK, Samarate -(VA) acl 19;

- CERMET, Bologna, acl 52;

- DELO Services . s.r.l., Fizzonasco Pieve (MI), acl 121 ;

- EMIT LAS, Milan, acl 24;

- FASINTERNATIONAL, Milan, acl 128;

- GAMMA MISURE, Calenzano - (FI), acl 56;

- IMQ, Milan acl 21.

- METRA, Augusta (SR), acl 188.

- METRIX, S. Stefano Cisquina (AG), acl 171.

- NEMKO, 4 Biassono (MI) acl 42;

- QUALITY SERVICE, Venice, acl 112;

- SIMAV S. Maurizio Can. - TO) acl 64.

- SIMAV. Milan, acl 04;

- TECHNOSKY, Rome , acl 15;

- TELECOM, Turin, acl 72;

- TESI, Subbiano (AR), acl 101;

- TRESCAL, Zola Predosa (BO) acl 174.

\section{Travelling Standards}

The transportable standards for the comparison were three Tinsley $\bmod 3504 \mathrm{C} 1 \mathrm{~m} \Omega, 10 \mathrm{~m} \Omega$ and $100 \mathrm{~m} \Omega$ four terminal standard resistors to be calibrated respectively at $5 \mathrm{~A}$ and $20 \mathrm{~A}(1 \mathrm{~m} \Omega), 1 \mathrm{~A}$ and $5 \mathrm{~A}(10 \mathrm{~m} \Omega), 0.1 \mathrm{~A}$ and $1 \mathrm{~A}$. $(100 \mathrm{~m} \Omega)$. View of the model of these standards is given in Figure 1 and 2 . The travelling package included

Corresponding author: f.galliana@inrim.it 
also a thermal collector for each resistor (Figure 1) to be inserted in the resistors to be in thermal contact

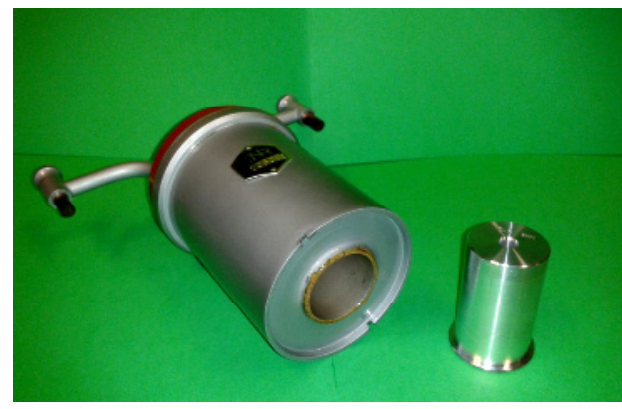

Figure. 1. Tinsley mod $3504 \mathrm{C}$ resistor with the thermal collector to put inside.

with them and suitable to measure their temperature and a glass beaker to be filled with $500 \mathrm{ml}$ of mineral oil. The measurements had to be carried out placing resistors inside it and acquiring the regime temperature of the thermal collector inside the resistor (Figure 2).

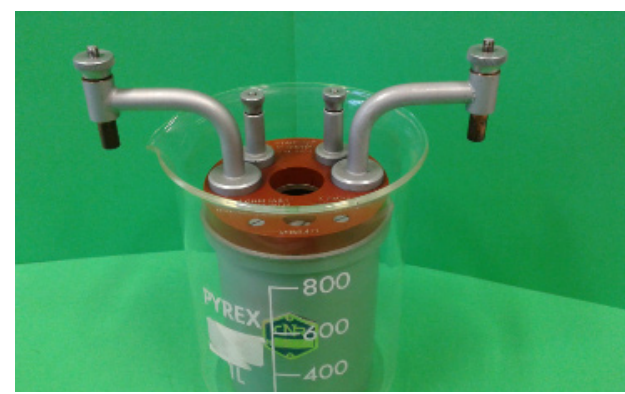

Figure 2. Tinsley mod 3504C resistor with the thermal collector inside the glass beaker.

Before the start of the comparison the three resistors were characterized evaluating their power and temperature coefficients. These measurements were made by the Low Resistances Calibration Laboratory of INRIM and performed with a measurement method involving high precision standard resistors put in a thermo-regulated at $23 \pm 0.3){ }^{\circ} \mathrm{C}$ oil-bath, a high performance current comparator bridge and a high stability power supply [3, 4]. The travelling resistors were placed in another thermo-regulated oil-bath with similar characteristics. The evaluated temperature and power coefficients of the travelling resistor are reported in Table 1 and 2 .

Table 1. Temperature coefficients of the travelling resistors.

\begin{tabular}{|c|c|c|}
\hline Resistor & $\boldsymbol{\alpha}$ & $\boldsymbol{\beta}$ \\
\hline $1 \mathrm{~m} \Omega$ & $\cong 1.0 \times 10^{-5} /{ }^{\circ} \mathrm{C}$ & $\cong-6.0 \times 10^{-7} /{ }^{\circ} \mathrm{C}^{2}$ \\
\hline $10 \mathrm{~m} \Omega$ & $\cong 9.3 \times 10^{-6} /{ }^{\circ} \mathrm{C}$ & $\cong 5.5 \times 10^{-7} /{ }^{\circ} \mathrm{C}^{2}$ \\
\hline $100 \mathrm{~m} \Omega$ & $\cong-8.9 \times 10^{-6} /{ }^{\circ} \mathrm{C}$ & $\cong-4.7 \times 10^{-7} /{ }^{\circ} \mathrm{C}^{2}$ \\
\hline
\end{tabular}

Table 2. Power coefficients of the travelling resistors.

\begin{tabular}{|c|c|}
\hline Resistor & Power coeff \\
\hline $1 \mathrm{~m} \Omega$ & $\cong 4.1 .0 \times 10^{-8} / \mathrm{mW}$ \\
\hline $10 \mathrm{~m} \Omega$ & $\cong 3.7 \times 10^{-8} \mathrm{~mW}$ \\
\hline $100 \mathrm{~m} \Omega$ & $\cong-8.1 \times 10^{-8} / \mathrm{mW}$ \\
\hline
\end{tabular}

\subsection{Time drift of the travelling resistors}

The $1 \mathrm{~m} \Omega$ resistor showed a high stability following a $2^{\text {nd }}$ order polynomial drift of about $1.6 \times 10^{-8} /$ day and $3.0 \times 10^{-8} /$ day, respectively at $5 \mathrm{~A}$ and $20 \mathrm{~A}$ during the whole period in the INRIM measurements, with only a small rise in the middle period.

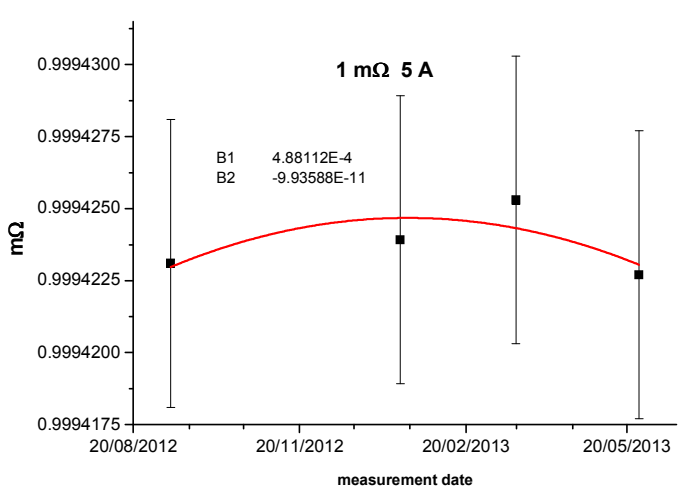

Figure 3. INRIM measurements $2^{\text {nd }}$ order polynomial fit of the $1 \mathrm{~m} \Omega$ resistor at $5 \mathrm{~A}$.

Also the $10 \mathrm{~m} \Omega$ resistor showed a high stability with a $2^{\text {nd }}$ order polynomial drift of about $3.8 \times 10^{-8} /$ day and $2.9 \times 10^{-8} /$ day, respectively at $1 \mathrm{~A}$ and $5 \mathrm{~A}$ during the whole period of the comparison in the INRIM measurements, with also in this case a small rise in the middle period at $1 \mathrm{~A}$, and smoothly decreasing at $5 \mathrm{~A}$.

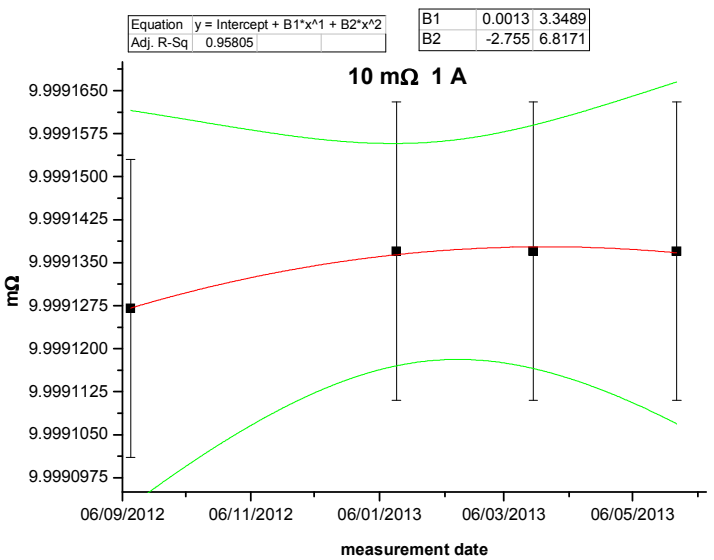

Figure 4. INRIM measurements $2^{\text {nd }}$ order polynomial fit of the $10 \mathrm{~m} \Omega$ resistor at $1 \mathrm{~A}$. 
Finally also the $100 \mathrm{~m} \Omega$ resistor showed a high stability with linear decreasing drift of about $4.4 \times 10^{-9} /$ day both at $0.1 \mathrm{~A}$ and $1 \mathrm{~A}$ during the whole period of the comparison in the INRIM measurements.

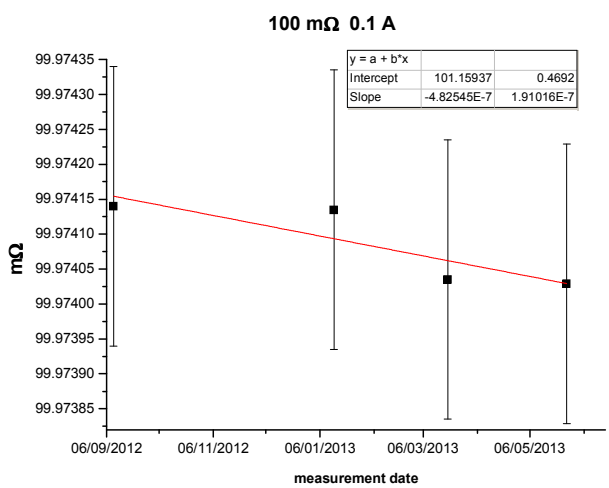

Figure 5. INRIM measurements linear fit on the $100 \mathrm{~m} \Omega$ resistor at $0.1 \mathrm{~A}$.

\subsection{Calibration modalities}

The Laboratories had to perform the calibration of the resistors in four-terminal configuration at the current values and time applications shown in Table 3 . If the adopted measurement method did not allow the choice of the measuring current, the applied current value and its application time had to be stated. Before starting the measurements it was suggested to wait two hours to stabilize the resistors in the oil. After performing the measurement at higher current, if was suggested to repeat the cycle after a waiting time of four hours. The reference ambient temperature of the laboratory had to be $23{ }^{\circ} \mathrm{C}$ and the Laboratory had to indicate the ambient temperature at the time of calibration. The Laboratory had to acquire also the temperature at the thermal regime reached for each measurement current. This temperature had to be measured at the thermal shaft junction of the resistor with a thermometer or a temperature sensor; these values allowed to correct at $23{ }^{\circ} \mathrm{C}$ the Laboratories measurements for a more correct evaluation of the compatibility with respect INRIM.

Table 3: Resistors under calibration along with currents and their application times.

\begin{tabular}{|c|c|c|}
\hline \multirow{2}{*}{ Resistor } & $\begin{array}{c}\text { Meas. } \\
\text { currents }\end{array}$ & $\begin{array}{c}\text { Application } \\
\text { times }\end{array}$ \\
\hline \multirow{2}{*}{$1 \mathrm{~m} \Omega$} & $5 \mathrm{~A}$ & $5 \mathrm{~min}$ \\
\cline { 2 - 3 } & $20 \mathrm{~A}$ & $15 \mathrm{~min}$ \\
\hline \multirow{2}{*}{$10 \mathrm{~m} \Omega$} & $1 \mathrm{~A}$ & $5 \mathrm{~min}$ \\
\cline { 2 - 3 } & $5 \mathrm{~A}$ & $15 \mathrm{~min}$ \\
\hline \multirow{2}{*}{$100 \mathrm{~m} \Omega$} & $0.1 \mathrm{~A}$ & $5 \mathrm{~min}$ \\
\cline { 2 - 3 } & $1 \mathrm{~A}$ & $15 \mathrm{~min}$ \\
\hline
\end{tabular}

\section{Measurement procedures}

The INRIM measurements were carried out with the previously mentioned measurement system involving high precision standard resistors and an high performance current comparator. The participating Secondary Laboratories measured the three standard resistors following their measurement procedures. In particular the involved measurement methods were:

- Direct reading with a digital multimeter (DMM), in particular for the $100 \mathrm{~m} \Omega$ standard at $0.1 \mathrm{~A}$. For the other measurement points this method didn't allow to perform the measurements at upper current values, so those measurements were considered as not accredited ones.

- Substitution method with a standard shunt or resistor connected in series with the resistor under calibration and supplied by a high stability current source (multifunction calibrator) and a DMM to measure the voltage drops on the standard and under calibration resistors.

- Volt-ampere-metric method with a high precision current source (multifunction calibrator) and a DMM to measure the voltage drop on the resistor under calibration.

\section{Analysis of the results}

To evaluate the measurement comparison the results of each measurement point were analyzed in the same way of $[5,6]$. The measurement values obtained by the Laboratories and by INRIM were defined respectively as: $m_{L a b} \pm u_{L a b}$ and $m_{I} \pm u_{I}$

where $m_{L a b}, u_{L a b}, m_{I}, u_{I}$ are respectively the measurement values and the standard uncertainties of the Laboratories and of INRIM. Both INRIM and the Laboratories gave their results along with the reached regime temperature at each measurement current, so that all the measurement values were reported at the temperature of $23.0{ }^{\circ} \mathrm{C}$, starting from their temperature coefficients. For $m_{I}$ were taken into account the interpolated values of INRIM measurements at the date of the measurements of the Laboratories. With this interpolation, an uncertainty due to possible errors due to the fitting operation was added to the INRIM uncertainty. A new measurand:

$$
y_{\text {Labr }}=\left(m_{\text {Lab }}-m_{I}\right) / m_{I}
$$

was defined for each measurement point whose relative standard uncertainty is:

$u_{y_{L a b, r}}^{2}=\frac{1}{m_{I}^{2}} \times\left[u_{L a b}^{2}+u_{I}^{2}-2 u_{L a b} u_{I} \times r\left(m_{L a b}, m_{I}\right)\right]$

where $r\left(m_{L a b}, m_{I}\right)$ is the correlation factor between the measurements of the Laboratories and those of INRIM, evaluated as the ratio between the type $\mathrm{B}$ component of the uncertainty with which INRIM calibrated the 
reference standards of the Laboratories and the uncertainty declared by the same Laboratories for the comparison. Finally, the normalized error $E n$ with respect to INRIM for each measurement point and for each Laboratory was evaluated as:

$E n=\frac{y_{L a b, r}}{U_{y_{L a b, r}}}$ where $U_{y L a b, r}=2 u_{y L a b, r} 2 \sigma$

\section{Measurement results}

In Figure 6, the normalized errors $E n$ for each participant Laboratory and for each resistor and measurement current are reported as an estimate of the compatibility degree with INRIM measurements.

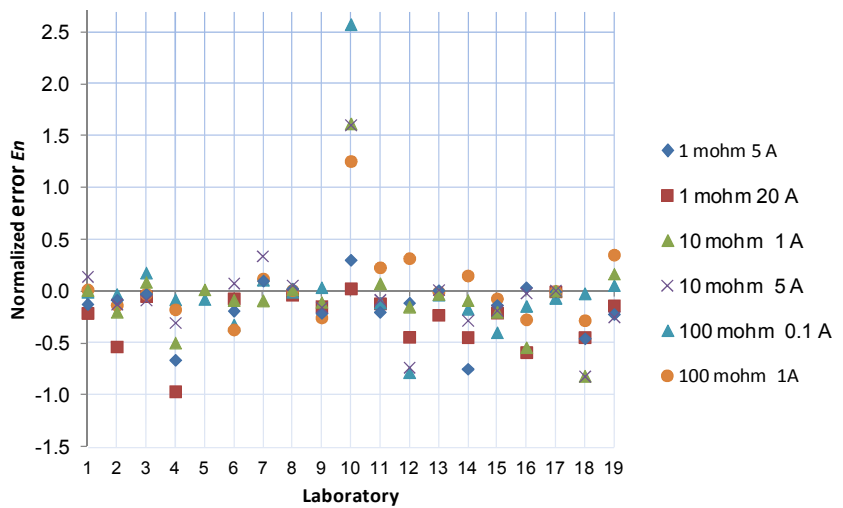

Figure 6. Laboratories normalized errors.

In Figures from 7 to 12 INRIM and Laboratories results corrected at $23{ }^{\circ} \mathrm{C}$ with their $2 \sigma$ uncertainties, are shown.

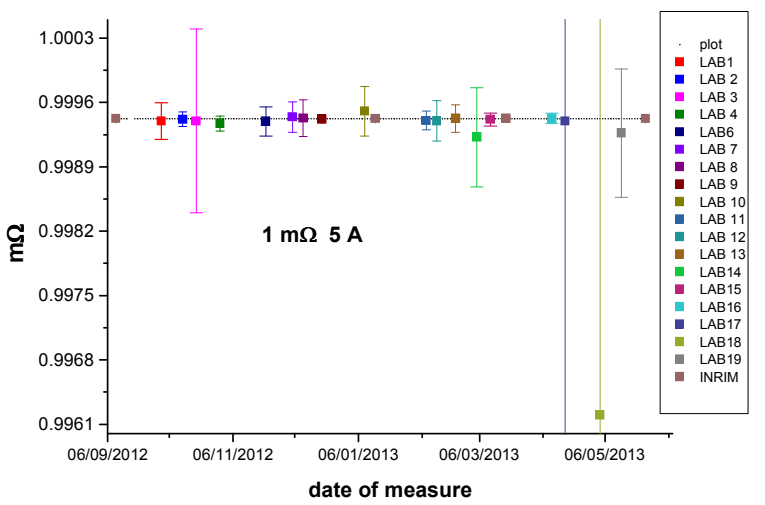

Figure 7. Results for the $1 \mathrm{~m} \Omega$ at $5 \mathrm{~A}$.

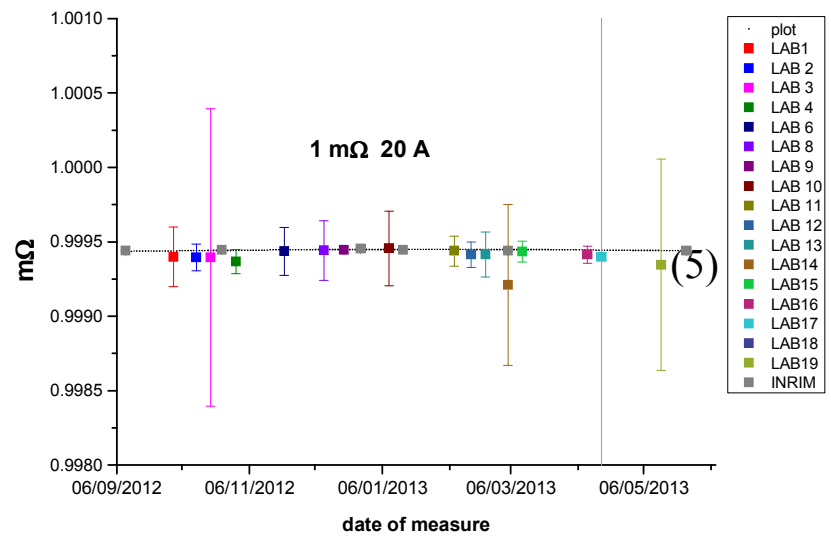

Figure 8. Results for the $1 \mathrm{~m} \Omega$ at $20 \mathrm{~A}$

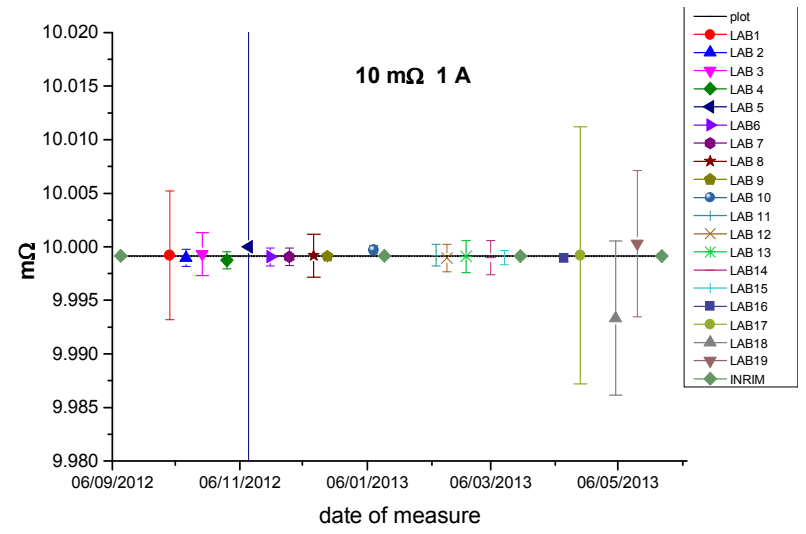

Figure 9. Results for the $10 \mathrm{~m} \Omega$ at $1 \mathrm{~A}$

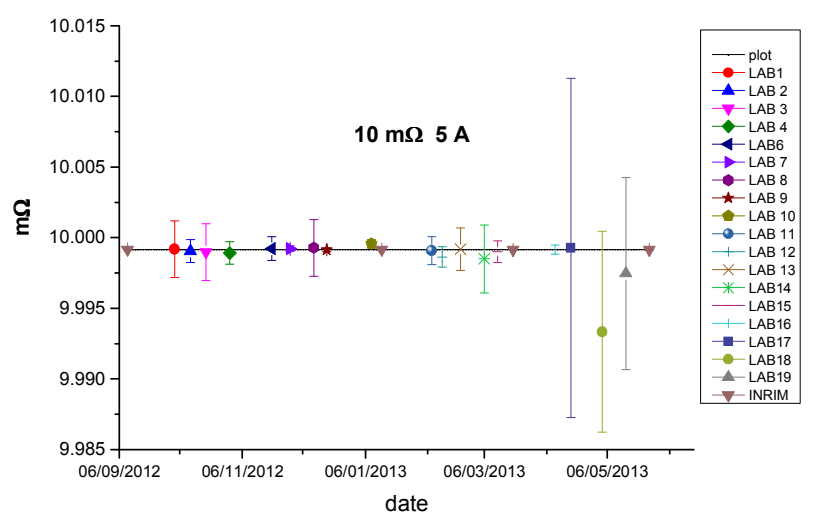

Figure 10. Results for the $10 \mathrm{~m} \Omega$ at $5 \mathrm{~A}$. Lab 5 did not make this measurement. 


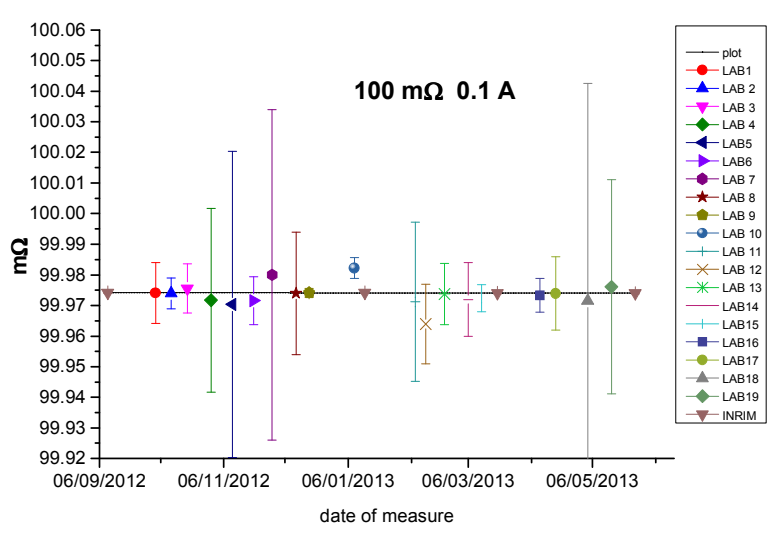

Figure 11. Results for the $100 \mathrm{~m} \Omega$ at $0.1 \mathrm{~A}$.

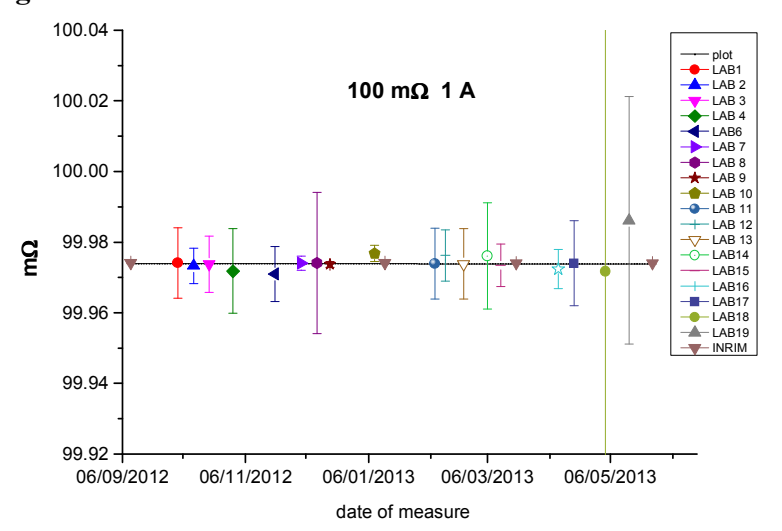

Figure 12. Results for the $100 \mathrm{~m} \Omega$ at $1 \mathrm{~A}$

\section{Discussion}

The condition that has to be satisfied for a correct traceability transfer from National Standards is that En $<|1|$. In figure 6 it is shown that that the majority of the measurement points satisfied this condition. It was accurately verified that all the measurements performed under accreditation satisfied the previous condition. In fact the measurements with $E_{n}>|1|$ were made in particular by a Laboratory that, although accredited for dc resistance, these fields are out of its accreditation field. Other border line $E_{n}$ values were from Laboratories that were not able to perform the measurements at the correct currents (for example reading directly with a DMM) but issued calibration certificates according to their accredited procedures. Considering all these conditions it can be concluded that the traceability transfer to Secondary Electrical Laboratories the field of low dc resistance is correctly assured.

\section{References}

1. ISO/IEC CD 17043 "Conformity assessment General requirements for proficiency testing" CASCO 05/2008, edition, 2008-03-14 previously ISO/IEC Guide 43-2 (1997).

2. EA-2/10. EA Policy for Participation in National and International Proficiency Testing Activities, rev.0, pp.20-20, (2001).
3. P. Mac Martin, L. Kusters, ," IEEE Trans. Instrum. Meas., Vol. IM-15, no. 5, pp:212-220, (1966).

4. P. Mac Martin, L. Kusters, IEEE Trans. Meas., Vol. IM-18, no. 4, pp.266-271, Dec. (1969).

5. F. Galliana, P.P. Capra, E. Gasparotto Measurement Vol. 42, pp. 1532-1540, (2009).

6. F. Galliana, P.P. Capra, E. Gasparotto, Measurement Vol. 45 no.3, pp. 615-621, (2012). 